\title{
Absolute differential cross sections for elastic scattering of electrons from pyrimidine
}

\author{
J. B. Maljković, ${ }^{1}$ A. R. Milosavljević, ${ }^{1 *}{ }^{*}$ F. Blanco, ${ }^{2}$ D. Šević, ${ }^{1}$ G. García, ${ }^{3}$ and B. P. Marinković ${ }^{1}$ \\ ${ }^{1}$ Laboratory for Atomic Collision Processes, Institute of Physics, Pregrevica 118, 11080 Belgrade, Serbia \\ ${ }^{2}$ Departamento de Física Atómica Molecular y Nuclear, Facultad de Ciencias Físicas, Universidad Complutense, Avda. Complutense s/n, \\ E-28040 Madrid, Spain \\ ${ }^{3}$ Instituto de Matemáticas y Física Fundamental, Consejo Superior de Investigaciones Científicas, Serrano 121, 28006 Madrid, Spain
}

(Received 26 March 2009; published 21 May 2009)

\begin{abstract}
Differential cross sections (DCSs) for elastic scattering of electrons from pyrimidine $\left(\mathrm{C}_{4} \mathrm{H}_{4} \mathrm{~N}_{2}\right)$ are presented for incident energies from 50 to $300 \mathrm{eV}$. The measurements were performed using a cross beam technique, for scattering angles from $20^{\circ}$ to $110^{\circ}$. The relative DCSs were measured as a function of both the angle and incident energy and the absolute DCSs were determined using the relative flow method. The calculations of electron interaction cross sections are based on a corrected form of the independent-atom method, known as the screen corrected additivity rule procedure and using an improved quasifree absorption model. Calculated results agree very well with the experiment.
\end{abstract}

DOI: 10.1103/PhysRevA.79.052706

PACS number(s): $34.80 . \mathrm{Bm}$

\section{INTRODUCTION}

Radiation damage in biomolecular systems has been the subject of extensive research work in the past few years. The major part of the energy deposited by ionizing radiation in condensed matter is channeled into the production of abundant secondary electrons [1-4]. To improve our understanding of the chain of reactions leading to DNA damage and postulate new reaction models, spectroscopic data and absolute cross-section values for electron impact on DNA and its constituents are needed. With this motivation, a number of studies both experimental and theoretical on electron interaction with building blocks of DNA molecule or their structural analogs have been reported in recent years.

Pyrimidine (Py), $\mathrm{C}_{4} \mathrm{H}_{4} \mathrm{~N}_{2}$, is a heterocyclic aromatic compound containing two nitrogen atoms at positions 1 and 3 of the six-member ring [5] (see Fig. 1). Three nucleobases found in nucleic acids, namely, cytosine $\left(\mathrm{C}_{4} \mathrm{H}_{5} \mathrm{~N}_{3} \mathrm{O}\right)$, thymine $\left(\mathrm{C}_{5} \mathrm{H}_{6} \mathrm{~N}_{2} \mathrm{O}_{2}\right)$, and uracil $\left(\mathrm{C}_{4} \mathrm{H}_{4} \mathrm{~N}_{2} \mathrm{O}_{2}\right)$ are pyrimidine derivatives. Because of its similarity to nucleotide ring system, Py has been considered as a model compound to investigate electron collisions from DNA constituents [6].

Although a huge number of papers have been reported in a few recent years devoted to the electron interaction with DNA constituents and their analogs, experimental results for Py are rather scarce. Palmer et al. [7] reported a study of electronic states of Py by means of vacuum ultraviolet (VUV) absorption, near-threshold electron energy-loss spectroscopy, and ab initio multireference configuration calculations. More recently, Levesque et al. [6] investigated electron-induced vibrational and electronic excitations of Py condensed on a thin film of solid argon held at $18 \mathrm{~K}$, for the incident energy range of 2-12 eV. However, according to our knowledge, there are no published measurements of the absolute differential cross sections (DCSs) for elastic electron scattering from Py molecule, neither generally for similar pyranouse compounds for the present energy range. Note

\footnotetext{
*vraz@phy.bg.ac.yu
}

that elastic DCSs for Py and pyrimidine nucleobases are expected to be very similar, both in shape and on the absolute scale, as has been shown recently for deoxyribose analog molecules [8]. On the other hand, calculations of elastic electron scattering by DNA (RNA) nucleobases have been performed recently by Možejko and Sanche [9]. They reported elastic DCSs for uracil, cytosine, guanine, adenine, and thymine, using independent-atom method (IAM) with a static-polarization model potential, for incident energies ranging from 50 to $4000 \mathrm{eV}$. The same authors also calculated total cross sections using the binary-encounter-Bethe model from the ionization threshold up to $5000 \mathrm{eV}$. Screening corrections not included in [9] are very significant at energies below $100 \mathrm{eV}$ for these species. These were included more recently by means of screen corrected additivity rule (SCAR) procedure in [10], where differential elastic and integrated (elastic and inelastic) cross sections were obtained for the five DNA/RNA bases in the $10 \mathrm{eV}-10 \mathrm{keV}$ energy range using an $a b$ initio quasifree atomic optical potential. Also, Winstead et al. [11] reported computed cross sections for elastic scattering of slow electrons by thymine and cytosine and by associated nucleosides, deoxythymidine, and deoxycytidine. For the isolated bases, they carried out calculations both with and without the inclusion of polarization effects.

In the present contribution, both experimental and theoretical results on elastic electron interaction with Py are reported. The absolute DCSs for elastic scattering of electrons from Py are obtained for the incident energies from 50 to 300 $\mathrm{eV}$ and in the angular range from $20^{\circ}$ to $110^{\circ}$. The relative cross sections were measured both as a function of the scat-

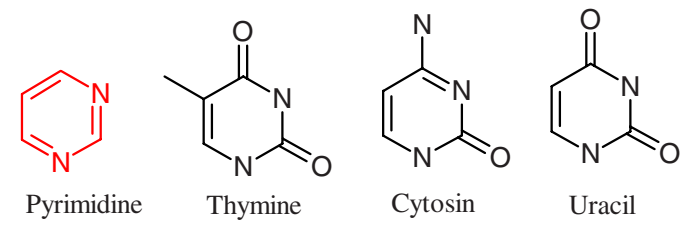

FIG. 1. (Color online) Schematic drawing of pyrimidine molecule and pyrimidine nucleobases. 
tering angle and incident electron energy and normalized to the absolute scale by using relative flow technique with $\mathrm{Ar}$ as a reference gas. Present calculations of molecular cross sections are based on a corrected form of the independent-atom method, known as the SCAR procedure. Furthermore, additional set of DCSs (SCARND in the following) is presented, which includes rotational excitations assuming a free electric dipole model and reduces the interference terms to ensure that integrated elastic values also satisfy the (corrected) additivity rule (see [8]). The calculated DSCs are in a very good agreement with the experimental results.

\section{EXPERIMENT}

The absolute DCSs for elastic electron scattering from Py were measured using a crossed electron-molecular-beam setup which has been described in detail previously $[8,12]$. Thus, we will only give a brief outline of the apparatus and the measurement procedure.

The experimental setup consists of an electron gun (hairpin electron source, up to about $1 \mu \mathrm{A}$ incident-beam current in the energy range from 20 to $500 \mathrm{eV}$ ), a double cylindrical mirror energy analyzer (DCMA), and a channel electron multiplier as a detector. All of these components are enclosed in a double $\mu$-metal shielded vacuum chamber. The incident electron beam is crossed perpendicularly by a molecular beam produced by a stainless still needle. The electron gun can be rotated around the needle in the angular range from about $-40^{\circ}$ to $120^{\circ}$. Scattered electrons are retarded and focused by a four-element cylindrical electrostatic lens into the DCMA, followed by a three-element lens to further focus transmitted beam into the channel multiplier. The base pressure of about $4 \times 10^{-7}$ mbar was obtained by a turbomolecular pump. The working pressure was usually in the range of $(2-5) \times 10^{-6}$ mbar and was checked for each experimental point. The uncertainty of the incident energy scale was determined to be less than $\pm 0.4 \mathrm{eV}$. The best energy resolution is limited by a thermal spread of primary electrons to about $0.5 \mathrm{eV}$. Therefore, the measured DCSs also include rotational and vibrational excitations, which should not contribute significantly for the present incident electron energy range. The angular resolution is better than $\pm 2^{\circ}$.

The anhydrous Py was purchased from Aldrich with a declared purity better than $99 \%$ and was used after several degassing cycles under vacuum, which were performed before each set of measurements. It was introduced into the scattering region from a glass container via a gas line system. Although Py possesses relatively high vapor pressure, the whole gas-handling system (sample container, pipes, and needle) was heated to provide stable experimental conditions and to improve the signal. Also, we have introduced a small temperature gradient in order to prevent the condensation of the sample vapor on the inner walls of the gas system. Sample container was kept at the temperature of about $50-60{ }^{\circ} \mathrm{C}$. The temperatures of the pipes and the needle were around $60{ }^{\circ} \mathrm{C}$ and $70{ }^{\circ} \mathrm{C}$, respectively. The background contributions, which were measured after redirecting the gas flow through the side leak, were typically below $15 \%$ and have been subtracted from the measured electron yields.
The relative DCSs were measured both as a function of the scattering angle and the incident electron energy [12]. In the latter case, the voltages of both the focusing part of the electron gun and the four-element entrance analyzer lens were adjusted as a function of the applied incident energy in order to obtain a constant incident electron beam and transmission function. For both types of relative measurements, the procedure was checked according to benchmark DCSs for elastic electron scattering by Ar [13-15]. The newly obtained DCSs for elastic electron scattering from Ar have been compared with previous results and small corrections of results for Py have been made. Benchmark DCSs were measured under the same experimental conditions.

The measured relative DCSs are normalized to the absolute scale according to the relative flow method [16,17], which is based on measurements of intensities for elastic scattering by a reference gas (Ar in the present case) and the gas under study, at a given incident electron energy $\left(E_{0}\right)$ and a scattering angle $(\theta)$. This technique demands experimental conditions where flows from the needle are almost identical for both gases (thus the beam profile in the collision region), which can be established with appropriate driving pressures behind the needle. It has been shown [17] that if these conditions are obtained, the absolute cross section for the gas under study (here Py) can be obtained according to the formula

$$
\operatorname{DCS}_{\mathrm{Py}}(E, \vartheta)=\operatorname{DCS}_{\mathrm{Ar}}(E, \vartheta) \frac{N_{\mathrm{Py}} F_{\mathrm{Ar}}}{N_{\mathrm{Ar}} F_{\mathrm{Py}}} \sqrt{\frac{M_{\mathrm{Ar}}}{M_{\mathrm{Py}}}},
$$

where $\operatorname{DCS}_{\mathrm{Py}}\left(E_{0}, \theta\right)$ and $\operatorname{DCS}_{\mathrm{Ar}}\left(E_{0}, \theta\right)$ are the absolute differential cross sections for elastic electron scattering from $\mathrm{Py}$ and reference gas, respectively; $N_{\mathrm{Py}}$ and $N_{\mathrm{Ar}}$ are the measured signal intensities; $F_{\mathrm{Py}}$ and $F_{\mathrm{Ar}}$ are the measured mass flow rates; and $M_{\mathrm{Py}}$ and $M_{\mathrm{Ar}}$ are the molecular weights.

A more detailed discussion on the present relative flow setup and procedure is given in our recent paper [8]. For the present experiment, the ratio of driving pressures (according to their gas-kinetic diameters) is determined to be $p_{\mathrm{Ar}}: p_{\mathrm{Py}}=1.7: 1$. During the measurements, variations in this ratio were within $\pm 12 \%$ of the defined value. These variations are considered to have negligible influence to the measured absolute DCS values $[8,18,19]$. The mass flow rates of the gases $\left(F_{\text {Ar }}\right.$ and $\left.F_{\text {Py }}\right)$ have been measured by closing the outlet to the gas chamber and recording the increase in the absolute pressure in the gas line behind the needle as a function of time (using an automated acquisition controlled by a PC LABVIEW program). Flow rates have been obtained from experimental curves, which were fitted by a least-squares method. In both cases (for Ar and Py) the increase in the pressure in time showed very strong linearity. For each experimental point, the background contributions (up to $10 \%$ ) were subtracted from the signal (note that in this case the background contains both $\mathrm{Ar}$ and Py).

The final set of the absolute DCSs is consistent with all three types of independently obtained results: the relative DCSs as a function of the scattering angle at fixed incident energy, the relative DCSs as a function of the incident energy at fixed scattering angle, and the relative flow measurements. 
Comparison of these three independent sets of results allows checking the experimental procedure and possible inconsistencies of reference cross sections.

The errors for the relative DCSs measured as a function of the scattering angle include statistical errors (0.1\%-5.5\%) according to Poisson's distribution and short-term stability errors $(1 \%-11 \%)$ according to discrepancy of repeated measurements at the same incident energy and scattering angle. The error of the relative angle-differential DCSs could be somewhat enlarged (up to 10\%-15\%) at small scattering angles (below $35^{\circ}$ ) due to corrections to the effective scattering volume, which were obtained according to benchmark DCSs measured for Ar.

The errors for the relative DCSs measured as a function of the incident energy include statistical errors $(0.4 \%-3 \%)$ and short-term stability errors $(8 \%-18 \%)$. Furthermore, since the relative energy-differential cross sections for Py are corrected (if needed) according to measured benchmark DCSs for Ar (see above), their overall error could be further increased up to about $20 \%$ due to an uncertainty of the reference relative DCS for Ar.

The errors for the absolute DCSs, obtained by relative flow technique, include error for reference DCSs for Ar [15], as well as errors of measured signal intensities and flow rates. Due to stable experimental conditions and high signal to background ratio, the uncertainty of the signal intensities and obtained flow rates are small. For all applied incident energies the error for the final signal intensities was less than $5 \%$ (except $10 \%$ for $150 \mathrm{eV}$ at $100^{\circ}$ ). Therefore, the overall error is dominantly defined by the error of the reference absolute DCSs. Reported errors for the reference absolute DCSs for $\operatorname{Ar}$ [15] are about $15 \%$. However, our recent investigation of existing elastic absolute DCSs for $\mathrm{Kr}$ [8] has shown that reported absolute values at the same electron energy and scattering angle, independently measured by different authors, could be considerably different. Furthermore, most of the authors estimated the overall error for the absolute DCSs to be about 20\%. Therefore, we believe that $20 \%$ uncertainty of the absolute elastic DCSs for Ar, which have been used as reference cross sections in the present work, is more reliable. The latter thus defines a minimal uncertainty of our results, while the overall error of the present absolute elastic DCSs for Py is estimated to be up to $25 \%$.

\section{CALCULATIONS}

Present calculations of molecular cross sections are based on a corrected form of the IAM, known as the SCAR procedure, with an improved quasifree absorption model potential, which includes relativistic and many-body effects, as well as inelastic processes. The same theoretical method has been already used in our recent work on deoxyribose analog molecules (see [8] and references therein), where an excellent agreement with experimental results has been obtained in the present angular and energy range, considering the shape of both angular and energy dependences of elastic DCSs, as well as their absolute values. The SCAR procedure has been described in detail previously [20,21]. For integrated cross sections, the role of SCAR correction to the standard IAM procedure is reducing the values obtained from the standard additivity rule to account for geometrical overlapping of atomic cross sections. The main effect to the shape of differential cross sections is a reduction in the forward scattering in favor of that at larger angles due to multiple scattering inside the molecule. Both effects are significant for present molecular sizes at energies below $100 \mathrm{eV}$.

The SCAR method ignores the rotational and vibrational excitations and considers only those inelastic processes arising from electronic excitation. Although this restriction is not significant in general for the relatively high energies (as used in the present work), in the case of molecules with a relatively high permanent dipole moment, rotational excitation becomes more important (see the recently published papers on the water [22] and 3HTHF [8] molecules). The dipole moment for Py has been reported to be about 2.3 D (see [23] and references therein). In the present work, we also introduce one more set of calculated DCSs where a previously applied procedure [22] has been used to account for this effect (SCARND procedure in the following). The method consists of the calculation of the rotational excitation cross section for a free electric dipole by assuming that the energy transferred is low enough, in comparison with the incident energy, to validate the first Born approximation. In these conditions, we have calculated an average rotational excitation cross section $J \rightarrow J^{\prime}$ for Py at $300 \mathrm{~K}$ by weighing the population of the $J$ rotational quantum number at that temperature and estimating the average excitation energy from the corresponding rotational constants. The most important effect of this correction is the increase in the absolute value of the cross section, which is significant at low incident energies and small scattering angles. Note, finally, that the SCARND method also includes a procedure where interference terms were normalized (reduced) as much as necessary to ensure that the integrated elastic values also satisfy the (corrected) additivity rule. This is as an attempt to solve a general problem of the IAM procedures when applied to differential calculations, which is an overestimation of the resulting interference contributions at small angles. The reliability of this normalization procedure has been discussed in detail in our previous paper [8] but it should be noted here that its main effect is an appreciable reduction in the differential elastic values at small angles below about $20^{\circ}$, while generally only small deviations from SCAR calculations are seen at higher angles.

\section{RESULTS AND DISCUSSION}

The absolute DCSs for elastic scattering of electrons from pyrimidine molecule have been experimentally obtained using relative flow technique and $\mathrm{Ar}$ as a reference gas. Relative flow measurements were performed for the incident electron energies of $50,100,150,200,250$, and $300 \mathrm{eV}$, at several scattering angles. Only for the incident energies of 70 and $120 \mathrm{eV}$ the absolute DCSs have been obtained according to the normalized relative DCSs independently measured as a function of the incident energy at the fixed scattering angles of $50^{\circ}$ and $110^{\circ}$. The accuracy of the final absolute DCS values depends on the used reference cross-section data 
TABLE I. Experimentally obtained differential cross sections for elastic electron scattering from pyrimidine, in units of $10^{-20} \mathrm{~m}^{2} \mathrm{sr}^{-1}$, as a function of scattering angle and incident electron energy. The absolute errors of relative cross sections (statistical, short-term stability, and uncertainty of the effective scattering volume) in the last significant digits are given in parentheses. The errors of the absolute cross sections are estimated to be up to $25 \%$.

\begin{tabular}{|c|c|c|c|c|c|c|c|c|}
\hline \multirow{2}{*}{$\begin{array}{l}\text { Scattering angle } \\
\text { (deg) }\end{array}$} & \multicolumn{8}{|c|}{ Electron energy $(\mathrm{eV})$} \\
\hline & 50 & 70 & 100 & 120 & 150 & 200 & 250 & 300 \\
\hline 20 & & & & & & & & $1.86(25)$ \\
\hline 25 & & & & & & & & $0.87(11)$ \\
\hline 30 & $1.423(93)$ & $1.45(13)$ & $1.839(65)$ & $1.45(13)$ & $1.40(22)$ & $1.05(21)$ & $0.457(41)$ & $0.682(84)$ \\
\hline 35 & $1.013(66)$ & $1.19(11)$ & $1.247(44)$ & $0.837(75)$ & $0.625(28)$ & $0.630(66)$ & $0.402(17)$ & $0.637(76)$ \\
\hline 40 & $0.925(61)$ & $0.915(83)$ & $0.737(26)$ & $0.467(42)$ & $0.424(19)$ & $0.526(17)$ & $0.322(32)$ & $0.426(53)$ \\
\hline 45 & $0.838(55)$ & $0.624(56)$ & $0.445(16)$ & $0.376(34)$ & $0.421(19)$ & $0.425(25)$ & $0.217(22)$ & $0.243(30)$ \\
\hline 50 & $0.704(46)$ & $0.406(37)$ & $0.358(13)$ & $0.368(33)$ & $0.384(18)$ & $0.280(17)$ & $0.132(13)$ & $0.173(10)$ \\
\hline 55 & $0.510(34)$ & $0.287(26)$ & $0.343(12)$ & $0.336(30)$ & $0.294(14)$ & $0.139(15)$ & $0.0817(76)$ & $0.1453(88)$ \\
\hline 60 & $0.351(23)$ & $0.256(23)$ & $0.319(12)$ & $0.266(24)$ & $0.2060(96)$ & $0.111(17)$ & $0.0727(74)$ & $0.1278(78)$ \\
\hline 65 & $0.261(18)$ & $0.250(23)$ & $0.2694(98)$ & $0.195(18)$ & $0.1426(68)$ & $0.1087(42)$ & $0.0692(35)$ & $0.1060(66)$ \\
\hline 70 & $0.239(16)$ & $0.238(22)$ & $0.2133(79)$ & $0.147(13)$ & $0.1098(53)$ & $0.0964(38)$ & $0.0611(31)$ & $0.0929(59)$ \\
\hline 75 & $0.238(16)$ & $0.216(20)$ & $0.1660(62)$ & $0.116(11)$ & $0.0965(47)$ & $0.0941(38)$ & $0.0568(29)$ & $0.0813(52)$ \\
\hline 80 & $0.239(16)$ & $0.201(18)$ & $0.1379(52)$ & $0.1010(93)$ & $0.0961(47)$ & $0.0865(35)$ & $0.0483(26)$ & $0.0785(81)$ \\
\hline 85 & $0.244(16)$ & $0.190(17)$ & $0.1297(49)$ & $0.1017(93)$ & $0.0941(46)$ & $0.0789(33)$ & $0.0445(24)$ & $0.0566(44)$ \\
\hline 90 & $0.253(17)$ & $0.177(16)$ & $0.1255(48)$ & $0.0996(91)$ & $0.0962(47)$ & $0.0792(33)$ & $0.0382(22)$ & $0.0496(55)$ \\
\hline 95 & $0.263(18)$ & $0.177(16)$ & $0.1259(48)$ & $0.1087(99)$ & $0.0961(47)$ & $0.0709(30)$ & $0.0314(19)$ & $0.0374(28)$ \\
\hline 100 & $0.269(18)$ & $0.179(16)$ & $0.1350(51)$ & $0.119(11)$ & $0.0968(47)$ & $0.0683(30)$ & $0.0272(17)$ & $0.0405(29)$ \\
\hline 105 & $0.295(25)$ & $0.190(17)$ & $0.1547(58)$ & $0.131(12)$ & $0.1037(50)$ & $0.0592(27)$ & $0.0257(16)$ & $0.0401(29)$ \\
\hline 110 & $0.329(32)$ & $0.216(20)$ & $0.1697(63)$ & $0.147(13)$ & $0.1065(51)$ & $0.0569(26)$ & $0.0274(17)$ & $0.0405(29)$ \\
\hline
\end{tabular}

set for Ar. Unfortunately, according to our knowledge, there are only few papers that report independently measured absolute elastic DCSs for Ar in the energy range of interest for the present work $[14,15]$. Furthermore, only the results of Williams and Willis [15] published for the incident electron energies between 20 and $400 \mathrm{eV}$ cover the whole energy range of the present work, thus allowing us to obtain a consistent set of reference DCSs for Py. It should be noted that when the absolute DCSs for Py at 50 and $100 \mathrm{eV}$ are derived using the reference DCSs for Ar published by Srivastava et al. [14], they differ only for about $0.5 \%$. The present experimental absolute elastic DCSs for Py are tabulated in Table I. Finally, to enable an estimate of renormalization of the present experimental results for Py to different possibly more accurate Ar DCSs, we indicate several values of absolute DCSs for Ar [15] used for normalization (in units of $10^{-20} \mathrm{~m}^{2} \mathrm{sr}^{-1}$ ) as follows: $\left(50 \mathrm{eV}, 40^{\circ}\right)-1.042$; $\left(100 \mathrm{eV}, \quad 40^{\circ}\right)-0.2492 ; \quad\left(150 \mathrm{eV}, \quad 40^{\circ}\right)-0.1610$; $\left(200 \mathrm{eV}, 50^{\circ}\right)-0.08764 ;\left(250 \mathrm{eV}, 50^{\circ}\right)-0.08232$; and $\left(300 \mathrm{eV}, 40^{\circ}\right)-0.1257$.

The experimental absolute angle-differential cross sections are presented in Fig. 2 (full circles). The DCS values obtained directly by relative flow measurements which were used for calibration are presented, as well (open stars). The relative normalized DCSs fit very well to the points obtained by relative flow, thus confirming consistency of the measurements. It is interesting to note that the angular dependence of elastic DCSs for Py appears to be similar to those for the deoxyribose analog molecules tetrahydrofuran (THF), 3HTHF, and tetrahydrofurfuryl alcohol (THFA) (see [8]). It is characterized by a small shoulder around $40^{\circ}-50^{\circ}$ and a broad minimum around $80^{\circ}-90^{\circ}$, with the latter being mostly pronounced at the energy of about $100 \mathrm{eV}$ and disappears at higher energies. Note that absolute values of DCSs for Py are also close to those for deoxyribose analogs. Therefore, it could be expected that elastically scattered electrons both from pyrimidine bases and sugar constituents of the DNA (RNA) backbone are similarly redistributed for the incident energies above about $50 \mathrm{eV}$.

Experimental results for Py are compared with present SCAR (full curve) and SCARND calculations (dashed curve), as well as with previous theoretical results for uracil obtained by IAM technique (dotted curve). As already confirmed previously for other molecules (see [8] and references therein), the SCAR calculations show remarkable agreement with the experimental cross sections, considering both the relative behavior and absolute values. However, in the present case for Py, a noticeable disagreement appears for the lowest energies (50 and $70 \mathrm{eV}$ ) in the lower angular range below about $40^{\circ}-50^{\circ}$. We cannot give a full explanation for this. From one side, the possible experimental influence cannot be absolutely excluded since generally the electron beam spreads with decreasing the incident electron energy, thus increasing an uncertainty of reliable background subtraction and effective volume correction. Still, the DCSs measured for rare gases at these energies show a very good agreement 


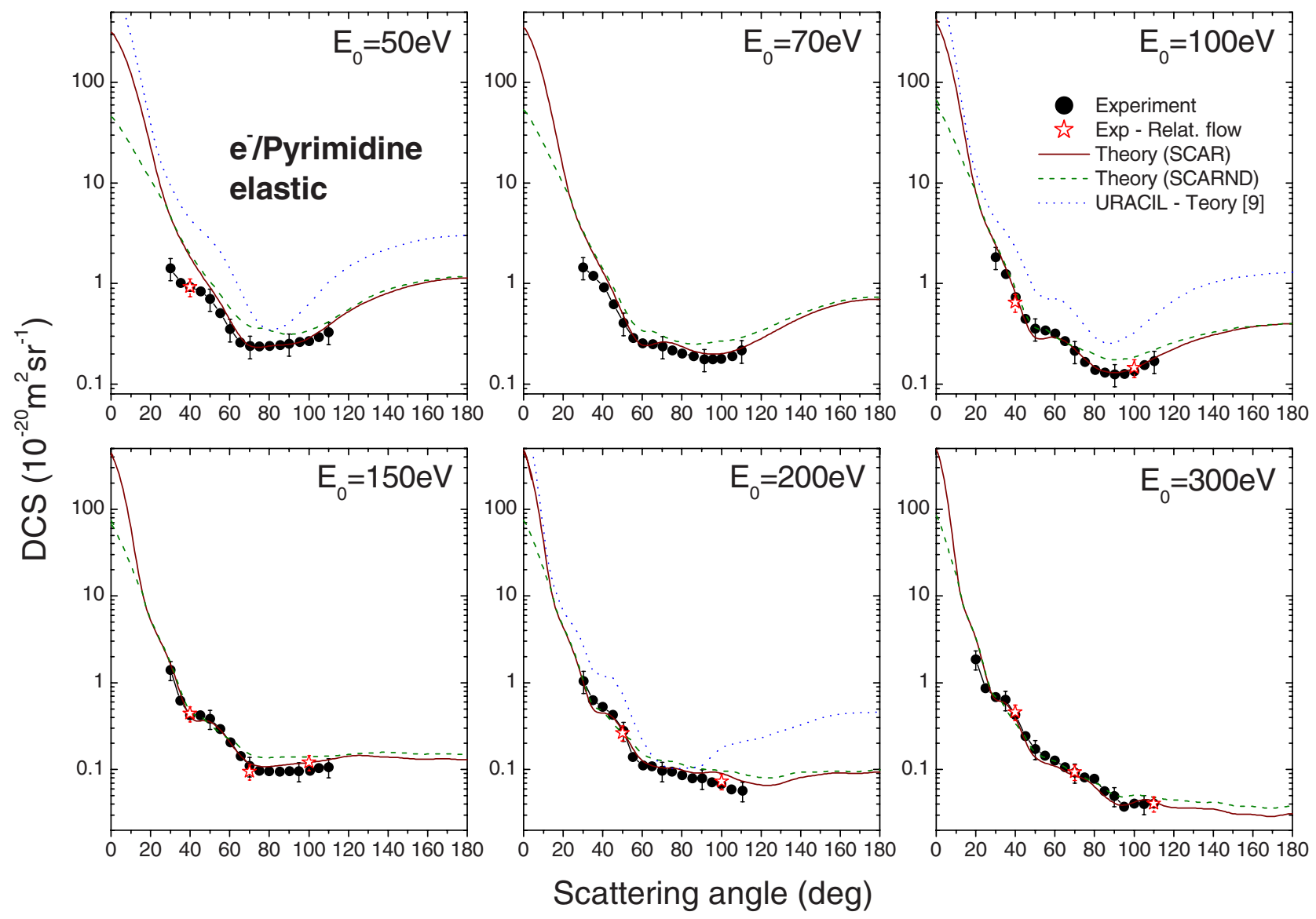

FIG. 2. (Color online) Angular dependence of absolute differential cross sections for elastic electron scattering from pyrimidine at different incident energies. Full circles represent absolute experimental differential cross. Open stars represent absolute values obtained by relative flow method. The SCAR calculations are presented by full line and the SCARND by dashed line. The previous theoretical results for uracil [9] are presented by dotted curve.

with previously published data. From another side, although the present theoretical method based on the independentatom model is expected to be less reliable at lower energies, the same method showed an excellent agreement with the experiment even down to $40 \mathrm{eV}$ for 3HTHF molecule [8]. Still, it should be noted that the dipole moment for Py is somewhat higher than for 3HTHF molecule, which could influence the SCAR procedure at low energies and small scattering angles (see [22]). The DCSs calculated using the SCARND procedure (dashed curves in Fig. 2), which includes rotational excitations assuming a free dipole model, are slightly higher around their minima in comparison with SCAR results (up to about 13\%) but are generally very close to SCAR results at higher incident energies. The differences are somewhat pronounced at low energies. Note that significant disagreement between SCAR and SCARND DCSs below about $20^{\circ}$ is dominantly induced by normalization procedure (SCARN, see [8]), which has been also applied in the SCARND calculations to reduce an overestimation of the resulting interference contributions at small angles. However, neither of these two procedures (SCAR nor SCARND) seems to give absolutely accurate DCSs at small scattering angles below about $20^{\circ}$, as we have recently discussed in detail [8].

It is interesting to note that the shoulder around $40^{\circ}$ as well as other maxima and minima observed in the differen- tial values arise from the interference terms in the IAM treatment, as we have checked that all of them disappear when no interference terms are included in the calculation. These interferences seem to be characteristic of the hexagonal ring structure, as they are very similar in size and position to those ones observed for benzene and several of its derivatives [24]. While similar oscillations are also present in the differential cross sections of tetrahydrofuran and its derivatives $[8,10]$ with pentagonal rings, those take place at somewhat different angles.

The IAM theoretical results for uracil [9] are published for the incident energies of 50, 100, and $200 \mathrm{eV}$, within the range of the present work. These DCSs generally show similar behavior in comparison with the present experimental and theoretical results for Py, considering the shape of the angular distributions. However, they are higher on the absolute scale. Although absolute cross sections for uracil are expected to be slightly higher than for Py considering the sizes of the molecules, we believe that the difference shown both on the absolute scale and in the DCS shape is mostly due to the applied theoretical method ignoring screening effects, as already shown previously $[8,10]$.

The dependence of absolute DCSs for elastic electron-Py scattering on the incident electron energy at fixed scattering angles of $50^{\circ}$ and $100^{\circ}$ is shown in Fig. 3. Experimental 


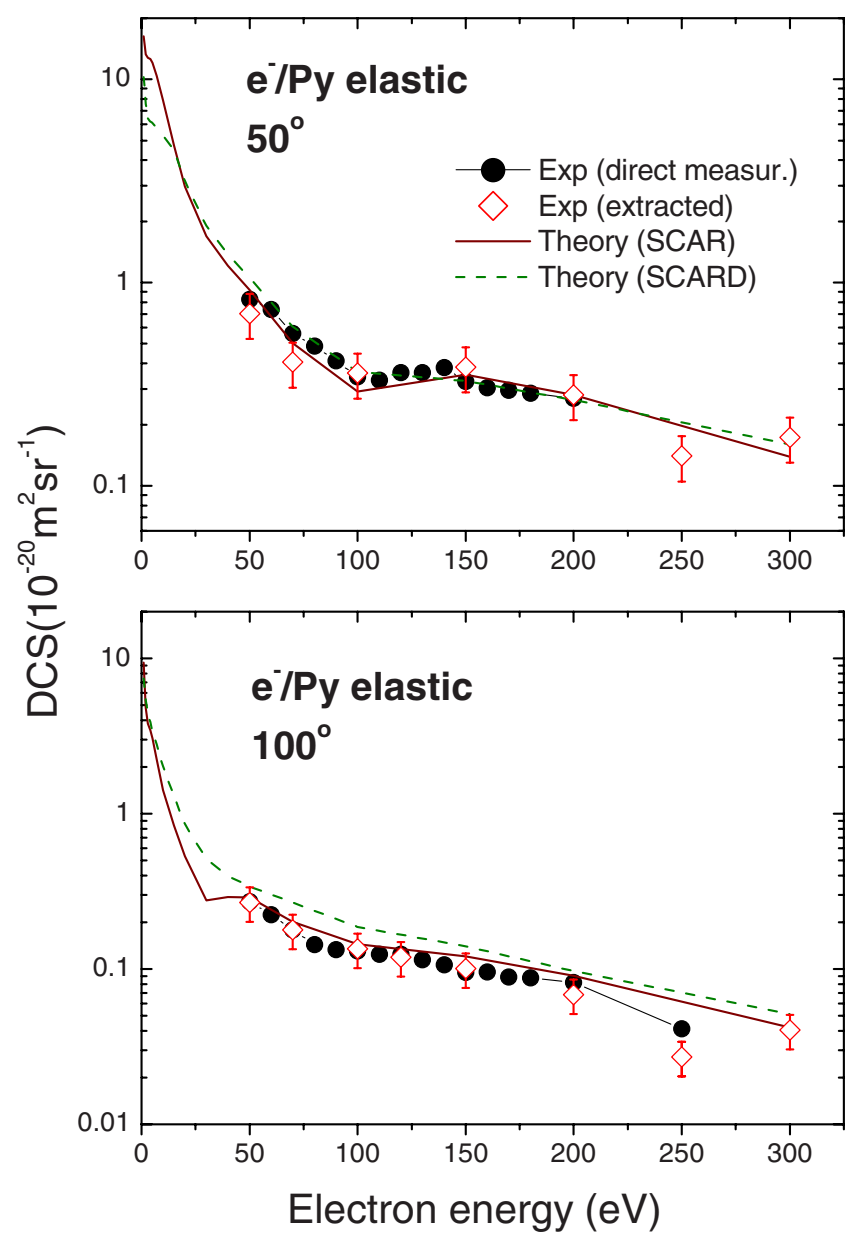

FIG. 3. (Color online) Energy dependence of absolute differential cross sections for elastic electron scattering from pyrimidine at the scattering angles of $50^{\circ}$ and $100^{\circ}$. Experimental differential cross sections (full circles) are normalized to the values extracted from the present absolute angle-differential cross sections (open diamonds). The SCAR calculations are presented by full line and SCARND by dashed line.

measurements (full circles) are normalized on the absolute scale according to DCSs values (open diamonds) which has been extracted from the absolute angular-dependent DCSs. For both angles $\left(50^{\circ}\right.$ and $\left.100^{\circ}\right)$, directly measured energydependent DCSs curves fit very well the points extracted from the absolute angle-differential cross sections. This confirms the consistency of the performed measurements.

Calculated energy-dependent DCSs obtained by SCAR (full line) and SCARND (dashed line) techniques are compared with experimental results. The SCAR theoretical curve compares very well with measurements, practically overlapping the experimental points in the whole measured energy range at both scattering angles. The only significant disagreement can be seen at the energy of $250 \mathrm{eV}$, where extracted experimental results both at $50^{\circ}$ and $100^{\circ}$ underestimate the theory. Thus we cannot totally exclude that this could be due to a systematic shift of reference cross sections for Ar. Nevertheless, it should be noted that independently measured relative energy-differential cross section at $100^{\circ}$ (full circles) agree well with the extracted points and also decreases at 250 $\mathrm{eV}$. Note that both experimental and theoretical results show a shallow minimum around $100 \mathrm{eV}$, for the energy dependence of DCS at the angle of $50^{\circ}$. The SCARND method gives slightly higher results on the absolute scale at $100^{\circ}$ (up to $20 \%$ ) but generally confirms both absolute value and the behavior of the energy-dependent cross sections.

\section{CONCLUSION}

The elastic scattering of electrons from pyrimidine molecule (Py) has been investigated both experimentally and theoretically. The present paper reports the absolute DCSs for elastic electron scattering by Py. The experimental absolute DCSs are tabulated for incident electron energies from 50 to $300 \mathrm{eV}$, in the angular range from $30^{\circ}$ to $110^{\circ}$ (from $20^{\circ}$ to $110^{\circ}$ at $300 \mathrm{eV}$ ). The measurements of the relative DCSs were performed as a function of both the scattering angle and the incident energy, using the cross-beam technique. The relative flow measurements, with $\mathrm{Ar}$ as a reference gas, provided absolute experimental points which have been used to normalize the relative DCSs. The calculations were performed using the SCAR procedure, with additional data sets to estimate rotational excitations and an overestimation of DCSs at small angles (SCARND). The calculated DCSs agree very well with the experimental points, considering both the shape and the absolute values. Finally, it should be noted that the present results suggest a very similar redistribution of medium energy $(40-500 \mathrm{eV})$ electrons upon elastic scattering by different DNA building blocks (doexyribose sugar or pyrimidine nucleobases).

The present results contribute to a fundamental understanding of electron interaction with biomolecules in the medium incident energy range. The tabulated absolute cross sections for Py can be used as starting parameters for energy deposition modeling in biologically relevant media and radiation damage research.

\section{ACKNOWLEDGMENTS}

We are very grateful to Professor P. Limão-Vieira from Lisbon who has initiated this work on pyrimidine molecule. We are also grateful to Dr. Pawel Možejko from Gdansk for sending us the calculated results for uracil in numerical form. This work was supported through Project No. 141011 financed by the Ministry of Science and Technological Development of Republic of Serbia and the Spanish Ministerio de Ciencia e Innovacion (Project No. FISI2006-00702). The present work was also motivated by research within COST Actions P9 "Radiation Damage in Biomolecular Systems" and CM0601 "Electron Controlled Chemical Lithography (ECCL)." 
[1] B. Boudaiffa, P. Cloutier, D. Hunting, M. A. Huels, and L. Sanche, Science 287, 1658 (2000).

[2] B. D. Michael and P. O. Neil, Science 287, 1603 (2000).

[3] H. Abdoul-Carime, P. C. Dugal, and L. Sanche, Radiat. Res. 153, 23 (2000).

[4] S. M. Pimblott, J. A. Laverne, and A. Mozumber, J. Phys. Chem. 100, 8595 (1996).

[5] T. L. Gilchrist, Heterocyclic Chemistry, 3rd ed. (Prentice Hall, New Jersey, 1997).

[6] P. L. Levesque, M. Michaud, and L. Sanche, J. Chem. Phys. 122, 094701 (2005).

[7] M. H. Palmer, I. C. Walker, M. F. Guest, and A. Hopkirk, Chem. Phys. 147, 19 (1990).

[8] A. R. Milosavljević, F. Blanco, J. B. Maljković, D. Šević, G. García, and B. P. Marinković, New J. Phys. 10, 103005 (2008).

[9] P. Možejko and L. Sanche, Radiat. Environ. Biophys. 42, 201 (2003).

[10] F. Blanco and G. García, Phys. Lett. A 360, 707 (2007).

[11] C. Winstead, V. McKoy, and S. d'Almeida Sanchez, J. Chem. Phys. 127, 085105 (2007).
[12] A. R. Milosavljević, S. Mandžukov, D. Ševic, I. Čadež, and B. P. Marinković, J. Phys. B 39, 609 (2006).

[13] D. Cvejanović and D. A. Crowe, J. Phys. B 30, 2873 (1997).

[14] S. K. Srivastava, H. Tanaka, A. Chutjian, and S. Trajmar, Phys. Rev. A 23, 2156 (1981).

[15] J. F. Williams and B. A. Willis, J. Phys. B 8, 1670 (1975).

[16] J. C. Nickel, C. Mott, I. Kanik, and D. C. McCollum, J. Phys. B 21, 1867 (1988).

[17] J. C. Nickel, P. V. Zetner, G. Shen, and S. Trajmar, J. Phys. E 22, 730 (1989).

[18] M. Dampc, A. R. Milosavljevic, I. Linert, B. P. Marinkovic, and M. Zubek, Phys. Rev. A 75, 042710 (2007).

[19] M. J. Allan, J. Phys. B 40, 3531 (2007).

[20] F. Blanco and G. García, Phys. Lett. A 317, 458 (2003).

[21] F. Blanco and G. García, Phys. Lett. A 330, 230 (2004).

[22] A. Munoz, J. C. Oller, F. Blanco, J. D. Gorfinkiel, P. LimaoVieira, and G. Garcia, Phys. Rev. A 76, 052707 (2007).

[23] J. Gao and K. Byun, Theor. Chem. Acc. 96, 151 (1997).

[24] H. Kato, M. C. Garcia, T. Asahina, T. Kuroda, M. Hoshino, C. Makochekanwa, H. Tanaka, F. Blanco, and G. García (unpublished). 